\title{
Life Analysis of Fan Spindle Bearing based on Grey Markov Algorithm
}

\author{
Han Zhang, Jianxin $\mathrm{Wu}^{*}$, Yiqiang Qiu, and Qiang Chen \\ College of Mechanical Engineering, Inner Mongolia University of Technology, Hohhot, 010051, China
}

\begin{abstract}
In order to improve the reliability and economic benefit of fan operation, a state estimation and residual life prediction method of fan spindle bearing based on grey Markov chain is proposed. Firstly, a grey distribution model of bearing wear condition of the wind turbine main shaft is established. Then, using the residual error correction (Markov prediction analysis method) and division of bearing wear state grade, which determines the state level interval limits, the main shaft bearing wear state transition probability is calculated and the state transition matrix of the Markov process is constructed. Finally, this method is used to calculate the life of the main shaft bearing of the wind turbine.
\end{abstract}

Keywords: spindle bearing; residual life; Markov; gray model

(Submitted on October 13, 2018; Revised on November 14, 2018; Accepted on December 21, 2018)

(C) 2019 Totem Publisher, Inc. All rights reserved.

\section{Introduction}

Due to global warming, energy shortage and environmental pollution problems are becoming increasingly serious, and many countries regard the development of renewable energy as an important strategic initiative. Wind power, for example, has become the third main power supply after thermal power and hydropower [1]. With the continuous development of society, the status of new energy in the energy industry has gradually increased, and wind power has received more and more attention. As fan motor assembly has increased, its structure has become more complex, and reliability problems such as bearing failure, gear, and other parts have become very prominent. The high loss efficiency of the wind turbine increases the cost of operation and maintenance and affects the economic benefit of the wind field. The momentum of such rapid development makes the performance of the wind turbine more significant. Because of the wind machine installed in wind energy resource rich regions, such as the coast of Gobi, the condition is very complicated. If one of the components fails, maintenance is very difficult. Ensuring fans with years of life in such conditions is a huge challenge of large-scale wind turbines, and solving the reliability problem of fan bearing parts is particularly important [2-4]. Wind power is mainly driven by the wind blade, and the whole system involves a large number of rotating components.

The bearings involved in the rotation of the wind turbine include the main bearings, the bearing of the generator, and the bearing of the gearbox, as shown in Figure 1 [4]. The bearings have many types, including cross roller bearings, cylindrical roller bearings, spherical roller bearings, and deep groove ball bearings. There are many technical difficulties for large yaw bearing assembly and blade spindle bearings. The localization of the bearing fan can improve the level of design and application of the domestic bearing industry, narrow the gap with a foreign advanced level, and promote domestic bearing industry development and technological progress. It can also reduce the cost of wind power and accelerate the development of new energy and renewable energy in China [2-6].

In each part of a large-scale wind turbine, the main bearing is the most important transmission system, and one of the main bearings is the most easily damaged part of large-scale wind turbine failure. Bearing damage will affect the entire wind turbine operation, and serious accidents caused by frequent bearing damage can even lead to wind machine downtime and increase the risk of fire damage. Wind fan bearing damage has seriously affected the stable operation of wind turbines

* Corresponding author.

E-mail address: 845872092@qq.com 
due to domestic airport shutdowns, as shown in Figure 2. It can result in severe economic losses, so life reliability analysis is very important [5].

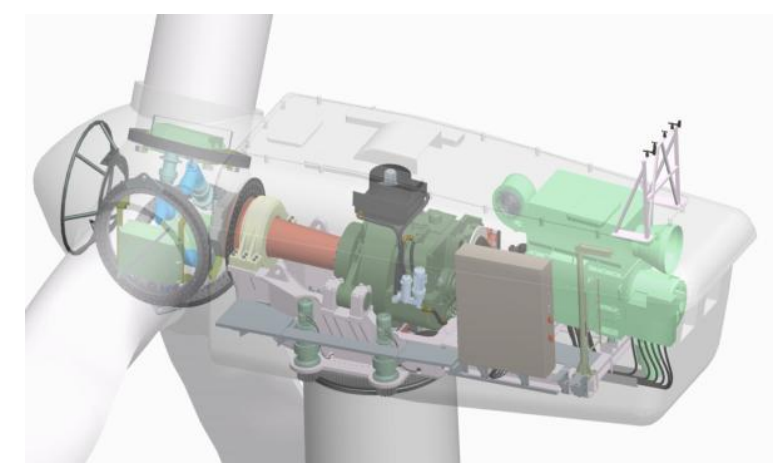

Figure 1. Wind generator bearing layout

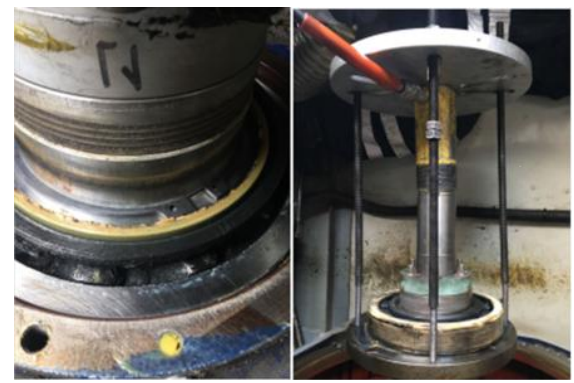

Figure 2. Picture of damage to spindle bearing

\section{The Main Research Content}

In this paper, we use a grey Markov prediction analysis method. Firstly, the grey model is established in the original data and the residuals are used for correction. Then, the residuals are regarded as Markov chain, the gamma data distribution model is established, and the maximum likelihood method is used to estimate the failure data of the parameters. Finally, the Markov process state transition matrix is constructed and the remaining life of the Chapman-Kolmogoroff equation is estimated.

Fan spindle bearing mainly adopts regular maintenance. A large amount of historical data has been accumulated during routine inspection and maintenance. Because the Markov chain is a mathematical statistical model based on time series, it can accurately describe the wear state of spindle bearing transfer in periodic inspection.

Various types of signals can be detected during the operation of wind turbines, including vibration signals related to bearing life prediction. Fan main shaft bearing is affected by height and volume, and we cannot observe the bearing failure problem, so monitoring vibration signal analysis is used to determine the actual operation condition of main shaft bearing and predict the equipment situation ahead of time.

As shown in Figure 3, the process diagram of calculating the life of the spindle bearing is simplified.

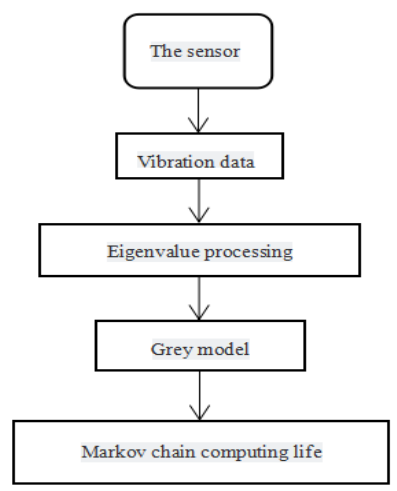

Figure 3. Summary of main content 


\subsection{The Method of Finding Eigenvalues}

The fault feature extraction methods of mechanical structures are mainly divided into two categories. The first class considers the relationship between variable patterns and is the realization of variable responses with patterns, such as the method of mutual information entropy and neural network method. The second class considers the relationship between variables through the contribution of variables of each group of fault modes to the fault classification, selects variables with large contributions, and deletes variables with small contributions. Typical methods are principal component analysis and wavelet packet analysis. Wavelet packet analysis is used in this paper.

Wavelet packet analysis is a new fault data analysis method based on non-stationary signal transformation that was presented 1989. Because the main shaft bearing fault signal of wind generators is mostly non-equilibrium signal or transient impact signal, the wavelet packet analysis method is applied.

Wavelet packet transform has the characteristics of guaranteeing signal integrity and orthogonality. Wavelet packet analysis is an extended method to decompose and reconstruct signals. After decomposition and reconstruction, the information of the signal is complete, and the signal components are neither redundant nor leaked. Figure 4 shows the fault vibration data of the main shaft bearing of a wind turbine in a wind field, reconstructed by the wavelet packet.

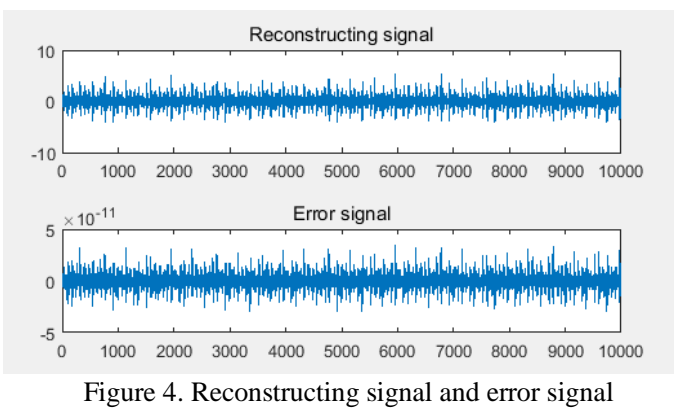

During the operation of the generator bearing, the surface damage point of the bearing element repeatedly impinges on the surface of the other elements that link to the neighborhood, which results in low-frequency vibration. This frequency is called bearing failure characteristic frequency.

In order to collect the original fault data, the fault feature information is separated and highlighted by the technical processing of the detected vibration signal. Figure 5 shows the original data of a bearing failure for a wind power machine field. The sampling frequency $f_{s}=12000$, and the sampling interval $T_{s}=1 / f_{s}$. The signal time domain waveform is shown in Figure 5.

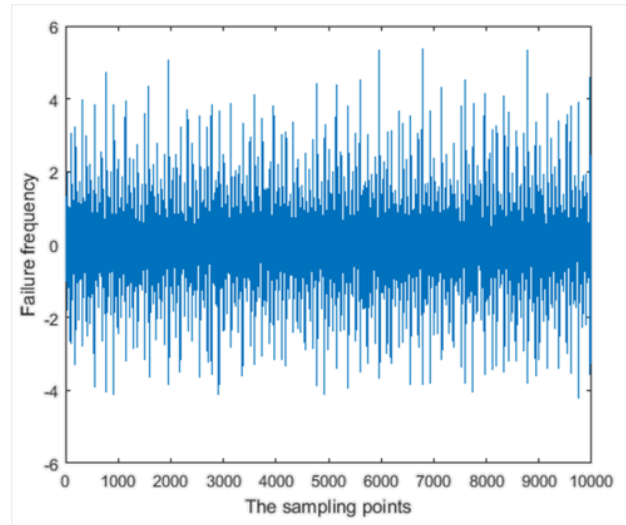

Figure 5. Raw data of a wind power machine field

The selection of wavelet function is diverse. Using different wavelet bases to analyze the same problem will have different effects. The Dn wavelet system is a classical wavelet function with many applications. Its characteristics are that with an increase in sequence number $\mathrm{N}$, the time domain support length and filter length become longer, the time locality worsens, the vanishing moment order and regularity increase, and the frequency domain localization improves. As shown in Figure 6, we will use the db10 wavelet, and the decomposition scale is 5 in this paper. 


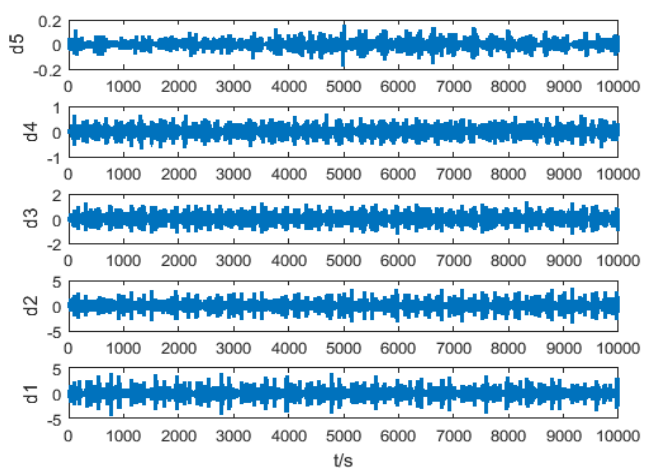

Figure 6. Five-layer wavelet packet decomposition

The envelope spectrum of the first layer of high frequency detail signal is shown in Figure 7.

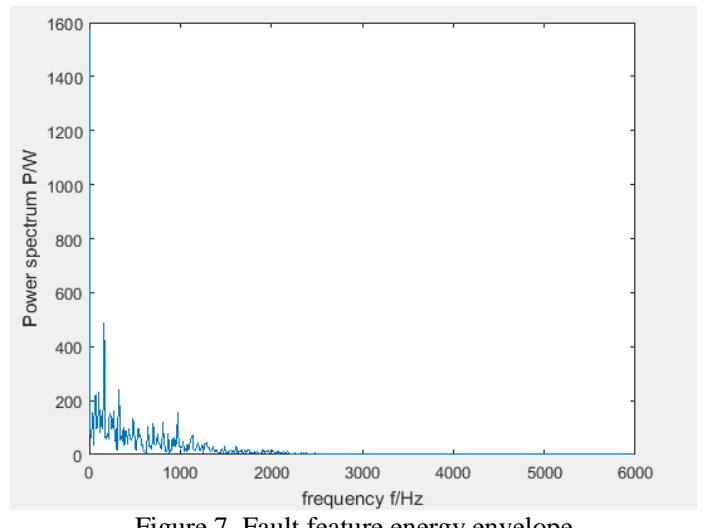

\subsection{Introduction to Grey Theory}

The grey system theory is a new subject that was founded in 1982. It regards some known information and unknown partial information, such as small sample, poor information, and uncertainty system, as the research objects. By generating some known information and extracting valuable information, a correct understanding of the operating rules of the system and the exact description are achieved and the [7] GM model is predicted. The advantages are that it does not require a large number of samples, the samples do not need regular distribution, and the computation workload is small. The quantitative analysis results are not inconsistent with the qualitative analysis results, which can be used for short-term, medium, and long-term prediction [7-8]. Due to many factors, such as the environment, it is very difficult for wind power airports to collect data. At the same time, the change of data is stochastic. Therefore, the use of the grey model is suitable for wind fields.

\subsection{The Establishment of Grey Model}

If the observed value of the eigenvalue of the system is $x^{(0)}=\left\{x_{1}^{(0)}, x_{2}^{(0)}, \cdots, x_{n}^{(0)}\right\}$, the steps of using this data sequence to establish an unbiased grey prediction model are as follows:

We use data accumulation to obtain $x^{(1)}$, and we know $x^{(1)}=\left\{x_{1}^{(1)}, x_{2}^{(1)}, x_{3}^{(1)} \cdots, x_{n}^{(1)}\right\}$.

- In order to achieve the purpose of data expansion.

- In order to determine the value of the data matrix $B$ and the specific process.

$$
\begin{gathered}
Y=\left(x^{(0)}(2), x^{(0)}(3), \cdots, x^{(0)}(n)\right)^{T} \\
B=\left[\begin{array}{cccc}
-\frac{1}{2}\left(x^{(1)}(2)+x^{(1)}(1)\right) & -\frac{1}{2}\left(x^{(1)}(3)+x^{(1)}(2)\right) & \cdots & -\frac{1}{2}\left(x^{(1)}(n)+x^{(1)}(n-1)\right) \\
1 & 1 & \cdots & 1
\end{array}\right]^{T}
\end{gathered}
$$


The parameter is estimated for first order linear differential equations by the least squares method $\hat{\alpha}$ and $\hat{\mu}$, because the GM model corresponds to the linear differential equation.

$$
\frac{\mathrm{d} x^{(1)}}{\mathrm{d} t}+\alpha x^{(1)}=\mu
$$

The parameters $\alpha$ and $\mu$ need to be identified, and it is necessary to estimate the parameters.

Differential equation:

$$
x^{(0)}(t)=\left[x^{(0)}(1)-\frac{\mu}{\alpha}\right] e^{-\alpha t}+\frac{\mu}{\alpha}
$$

Estimated by the least squares method $\hat{\alpha}$ and $\hat{\mu}$ :

$$
\left(\begin{array}{l}
\hat{\alpha} \\
\hat{\mu}
\end{array}\right)=\left(B B^{T}\right)^{-1} B^{T} Y
$$

The values of $B$ and $Y$ can be calculated by Equations (1) and (2).

$$
b=\ln \frac{2-\hat{\alpha}}{2+\hat{\alpha}}, A=\frac{2 \hat{\mu}}{2+\hat{\alpha}}
$$

The establishment of the original data sequence model is $\hat{x}^{(0)}(1)=x^{(0)}(1), \hat{x}^{(0)}(k+1)=A e^{b k}, k=1,2, \cdots, n$.

$$
\varepsilon^{(0)}(t)=\left|\hat{x}^{(0)}(t)-x^{(0)}(t)\right|, t=1,2, \cdots, n
$$

An accumulative sequence of the absolute values of the residuals is $\varepsilon^{(1)}(t)=\left\{\varepsilon^{(1)}(1), \varepsilon^{(1)}(2), \cdots, \varepsilon^{(1)}(n)\right\}$.

Now, we use a simple example to describe the data prediction of the grey model. Table 1 shows the bearing damage data for ten years in a fan field of the wind turbine factory. With the development of bearing maintenance technology in recent years, the annual number of bearing replacements of wind turbines is getting smaller and smaller. These data are applied to verify the established grey model.

Table 1. Bearing damage in a wind power plant

Table 1. Bearing damage in a wind power plant
\begin{tabular}{|c|c|c|c|c|c|}
\hline Year & 2008 & 2009 & 2010 & 2011 & 2012 \\
\hline Number & 66 & 63 & 65 & 59 & 58 \\
\hline \hline Year & 2013 & 2014 & 2015 & 2016 & 2017 \\
\hline Number & 65 & 58 & 57 & 61 & 63 \\
\hline
\end{tabular}

As shown in Figure 8, the red color is the prediction result and the predicted year is the next decade. The prediction curve shows a downward trend that it is in line with the actual operation of the wind power plant.

\subsection{Gamma Distribution and Parameter Estimation}

The wear of fan spindle bearing is a continuous degradation process. The degradation volume is stable in unit time. The independent and non-negative nature can be considered to obey the gamma distribution.

Distribution function:

$$
\tau(x)=\int_{0}^{ \pm \infty} \mu^{x-1} \mathrm{~d} \mu, x>0
$$

Probability density function: 


$$
f_{\alpha, \beta}(x)=\frac{\beta^{\alpha}}{\tau(\alpha)} x^{\alpha-1} e^{-\beta x}
$$

Where $\alpha$ is the shape parameter, $\beta$ is the scale parameter, and $f_{\alpha, \beta}(x)$ is the probability density function. The derivation of the partial derivatives of $\alpha, \beta$ are the estimated values $\hat{\alpha}, \hat{\beta}$.

$$
\ln L(\alpha, \beta)=\sum_{j=1}^{n}\left[\alpha \Delta t \ln \beta-\ln \tau(\alpha \Delta t)+(\alpha \Delta t-1) \ln \Delta x_{j}-\beta \Delta x_{j}\right]
$$

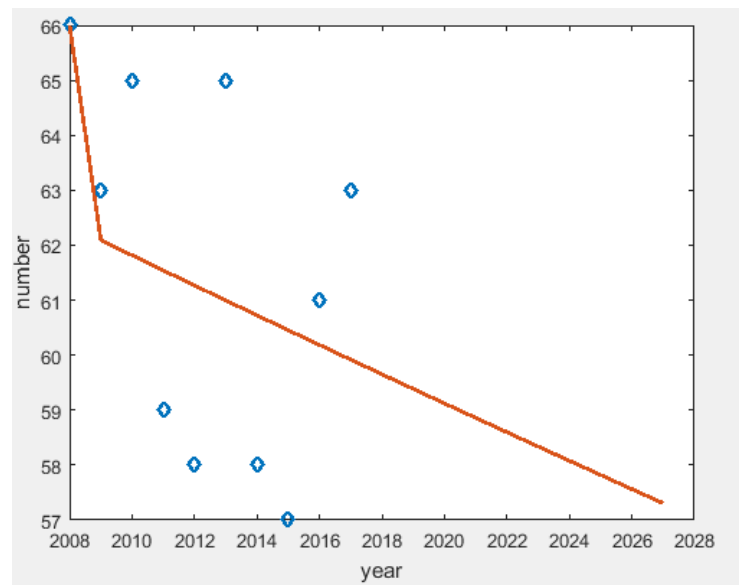

Figure 8. Prediction results of grey model

\subsection{Markov Chain Method}

Markov believed that the process of time and state was the discrete process called the Markov chain, which was the study of state transition probability. A large amount of historical data of fan spindle bearing are obtained mainly by regular maintenance and daily inspection, which can be regarded as the Markov chain based on time series. It can accurately describe the transfer process of spindle bearing damage state in periodic inspections [9-13].

State transition matrix:

$$
p=\left[\begin{array}{cccc}
p_{11} & p_{12} & \cdots & p_{1 m} \\
p_{21} & p_{22} & \cdots & p_{2 m} \\
\vdots & \vdots & \ddots & \vdots \\
p_{m 1} & p_{m 2} & \cdots & p_{m m}
\end{array}\right]
$$

Where the elements in Equation (11) are non-negative, the sum of each element is one, and $m$ is the divided state [14].

\subsection{State Assessment and Residual Life Prediction Steps}

The status assessment and residual life prediction of the main shaft bearing of the fan mainly include the following four steps [15-17]:

- A grey model is built on the original data, and the residual is used to modify it.

- On the basis of the grey model, the Markov chain is introduced to divide the wear state of the bearing.

- The gamma distribution model of data is set up, and the maximum likelihood method is used to estimate the parameters of the failure data.

- The structure of the Markov process state transition matrix is applied to estimate the remaining life of the ChapmanKolmogoroff equation. 


\section{Example Analysis}

According to the inspection and maintenance data of the fan gear box bearing, the wear condition of the fan gear box bearing can be divided into five states [17], which are normal (A), mild (B), moderate (C), severe (D), and invalid (D), as shown in Table 2.

Table 2. The bearing state of the main shaft of the wind turbine

\begin{tabular}{|c|c|c|}
\hline Wear state & State interval & State \\
\hline A & $(0 \sim 0.2)$ & Normal \\
\hline B & $(0.2 \sim 0.4)$ & Light \\
\hline C & $(0.4 \sim 0.7)$ & Moderate \\
\hline D & $(0.7 \sim 1)$ & Severe \\
\hline E & $>1$ & Need replace \\
\hline
\end{tabular}

According to the degenerate series of a certain type of fan spindle bearing in Table 3, the parameter estimation is carried out by the maximum likelihood method, and the shape parameter of the gamma distribution is obtained. $\alpha=2.497$, $\beta=0.2015$, and the probability density function of the degenerate increment is shown as follows:

$$
f(x)=\frac{0.2015^{2497}}{\Gamma(2.496)} x^{1.496} e^{-0.2015 x}
$$

According to the wear state limit of the spindle bearing given in Table 3, the state transfer matrix of the spindle bearing can be obtained as follows:

$$
p=\left[\begin{array}{ccccc}
0.7041 & 0.2951 & 0.0020 & 0.0008 & 0 \\
0 & 0.7887 & 0.2098 & 0.0015 & 0.0002 \\
0 & 0 & 0.6499 & 0.3500 & 0.0001 \\
0 & 0 & 0 & 0.5598 & 0.4400 \\
0 & 0 & 0 & 0 & 1
\end{array}\right]
$$

Table 3. Degradation data of the spindle bearing of wind turbine generator

\begin{tabular}{|c|c||c|c|}
\hline Number & Degenerate quantity & Number & Degenerate quantity \\
\hline 1 & 0.01 & 8 & 0.222 \\
\hline 2 & 0.023 & 9 & 0.224 \\
\hline 3 & 0.046 & 10 & 0.287 \\
\hline 4 & 0.066 & 11 & 0.234 \\
\hline 5 & 0.092 & 12 & 0.333 \\
\hline 6 & 0.137 & 13 & 0.352 \\
\hline 7 & 0.171 & 14 & 0.396 \\
\hline
\end{tabular}

Table 4 is the degenerate data of the main shaft bearing of a fan. The feasibility of the proposed method is verified by an example of the new running bearing. The starting state of the gear box bearing newly put into operation is A, where $\mathrm{A}=(1,0,0,0,0)$.

Table 4. Wear data of spindle bearing

\begin{tabular}{|c|c|c|}
\multicolumn{2}{|c|}{ Table 4. Wear data of spindle bearing } \\
\hline Check time & Wear amount & Wear state \\
\hline 0 & 0.014 & $\mathrm{~A}$ \\
\hline $1 \mathrm{~T}$ & 0.022 & $\mathrm{~A}$ \\
\hline $2 \mathrm{~T}$ & 0.045 & $\mathrm{~B}$ \\
\hline $3 \mathrm{~T}$ & 0.061 & $\mathrm{~B}$ \\
\hline $4 \mathrm{~T}$ & 0.093 & $\mathrm{~B}$ \\
\hline $5 \mathrm{~T}$ & 0.134 & $\mathrm{~B}$ \\
\hline $6 \mathrm{~T}$ & 0.168 & $\mathrm{~B}$ \\
\hline $7 \mathrm{~T}$ & 0.192 & $\mathrm{~B}$ \\
\hline
\end{tabular}

The results are evaluated in Table 5. It can be seen from Table 4 that the spindle bearing is in state after running $2 \mathrm{~T}$, B is still in state when running $7 \mathrm{~T}$, and there is no state of transfer during the period. The prediction results are in agreement with the actual degenerate state of the spindle bearing in Table 3, and the feasibility of this method is verified. 
Table 5. Wear state prediction of spindle bearing

\begin{tabular}{|c|c|c|c|c|c|c|}
\hline Inspect time & $\mathrm{A}$ & $\mathrm{B}$ & $\mathrm{C}$ & $\mathrm{D}$ & $\mathrm{E}$ & Abrasion state \\
\hline $\mathrm{T}$ & 0.702 & 0.2952 & 0.0020 & 0.0006 & 0 & $\mathrm{~A}$ \\
\hline $2 \mathrm{~T}$ & 0.4958 & 0.4406 & 0.0648 & 0.0017 & 0.0004 & $\mathrm{~B}$ \\
\hline $3 \mathrm{~T}$ & 0.3497 & 0.4942 & 0.1354 & 0.0243 & 0.0011 & $\mathrm{~B}$ \\
\hline$\vdots$ & $\vdots$ & $\vdots$ & $\vdots$ & $\vdots$ & $\vdots$ & $\vdots$ \\
\hline $9 \mathrm{~T}$ & 0.0425 & 0.2643 & 0.2194 & 0.1833 & 0.2973 & $\mathrm{E}$ \\
\hline
\end{tabular}

For the degenerate state of the spindle bearing at $2 \mathrm{~T}, \mathrm{~B}$ represents evaluating the starting state vector representation $\mathrm{A}=$ $(0,1,0,0,0)$. The residual life predictions of spindle bearing are shown in Table 5. The data in Table 6 show that the spindle bearing is always in the form of $3 \mathrm{~T}$ to $7 \mathrm{~T}$. Because the bearing has worked for $8 \mathrm{~T}$, the degradation is more serious, and the speed of degradation is faster. The transfer between the wear states is more rapid, and the spindle bearing state is within one inspection period of B to E. If the bearing failure is judged at this time, the remaining life of the bearing $T_{r}=6 \mathrm{~T}$.

Table 6. Residual life prediction

\begin{tabular}{|c|c|c|c|c|c|}
\hline state & A & B & C & D & E \\
\hline The number of Steps & 9 & 6 & 4 & 2 & 0 \\
\hline Residual life $T_{r}$ & $9 \mathrm{~T}$ & $6 \mathrm{~T}$ & $4 \mathrm{~T}$ & $2 \mathrm{~T}$ & 0 \\
\hline
\end{tabular}

The state transfer process corresponding to the starting state of the bearing is shown in Figures 9 and 10. The analysis shows that the bearing is in the process of the starting state to the failure state.

The predicted value of the distribution of the state is increasing. Two adjacent steps $\mathrm{E}$ of the difference between predicted values of state distribution probability is also increasing.

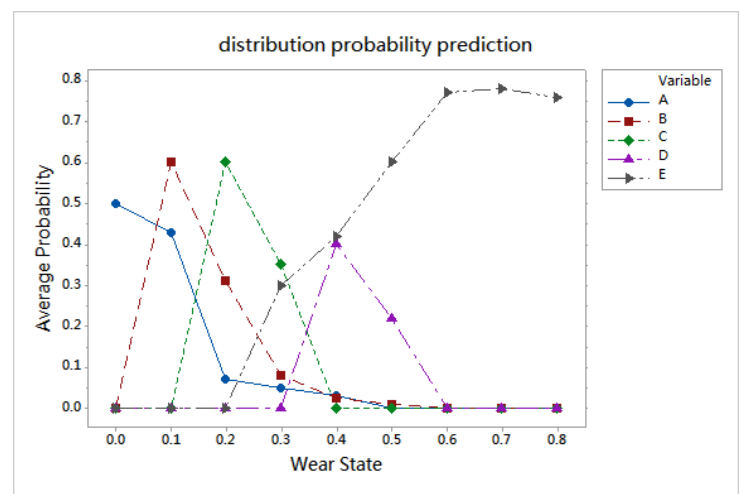

Figure 9. Distribution probability prediction

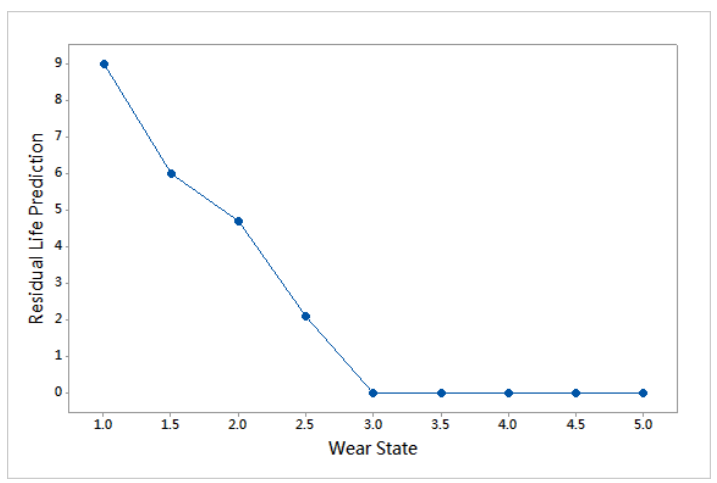

Figure 10. Residual life prediction

It indicates that the wear of spindle bearings is a gradual degradation process and the speed of degradation is accelerating. Spindle bearings A, B, C, D, and E correspond respectively to state transition step numbers 9T, 6T, 4T, 2T, and $0 \mathrm{~T}$, which indicates that the residual life of the spindle bearing corresponding to different initial states is good.

The corresponding residual life is long; otherwise, the residual life is short, which conforms to the objective laws of spindle bearing degradation. 


\section{Conclusions}

A state estimation and residual life prediction method of fan spindle bearing based on grey Markov chain is presented in this paper. This method reduces the dependence on a large number of monitoring data and does not need to install online monitoring equipment. The simulation results show the effectiveness of the method in determining the wear and residual life of the spindle bearing. Through the implementation of the proposed maintenance proposal, the number of blower outage accidents caused by the failure of the spindle bearing is reduced and the reliability of the fan operation is improved.

The reliability analysis for wind generators is still in its infancy, and potential theory and new tools are also needed to recognize the mechanism and improve the reliability.

\section{References}

1. B. Chen, "Analysis and Research on Fatigue Failure of Wind Turbine Main Shaft Bearings?" in 2012 Annual Proceedings of Wind Energy Equipment Branch of China Agricultural Machinery Industry Association, 2012

2. J. Hang, "Overview of Condition Monitoring and Fault Diagnosis Technology for Wind Power Generation Systems (English)," Journal of Electrical Engineering, Vol. 28, No. 4, pp. 261-271, 2013

3. X. H. Jin, Y. Sun, and Z. J. Zhai, "Research on the Prediction Method of Bearing Residual Life based on UKF," Journal of Instrument and Measurement, Vol. 37, No. 9, pp. 2036-2043, September 2016

4. X. T. Xia, "Performance Stability and Reliability Analysis of Rolling Bearings," Chinese Journal of Scientific Instrument, Vol. 38, No. 6, pp. 1421-1431, June 2017

5. X. D. Li, W. Liu, and S. H. Zhang, "Overview of Rolling Contact Fatigue Life Prediction Methods," Journal of Aviation Precision Manufacturing Technology, Vol. 53, No. 3, pp. 1-4, March 2017

6. R. Yin, "Discussion of Time-Dependent Reliability Analysis for Long-Term Bearing Capacity of Rock Tunnel Linings," Modern Tunnelling Technology, Vol. 53, No. 3, pp. 68-73, 2016

7. L. Li and H. Zhao, "Incipient Bearing Fault Diagnosis based on MCKD-EMD for Wind Turbine," Electric Power Automation Equipment, Vol. 37, No. 2, pp. 29-36, February 2017

8. D. Banjevic and A. K. S. Jardine, "Calculation of Reliability Function and Remaining Useful Life for a Markov Failure Time Process," IMA Journal of Management Mathematics, Vol. 17, No. 2, pp. 115-130, February 2006

9. P. Baruah and R. B. Chinnam, "Autonomous Diagnostics and Prognostics through Competitive Learning Driven HMM-based Clustering," in Proceedings of IEEE International Joint Conference on Neural Networks, Vol. 4, pp. 2466-2471, 2003

10. F. Camci and R. B. Chinnam, "Health-State Estimation and Prognostics in Machining Processes," IEEE Transactions on Automation Science and Engineering, Vol. 7, No. 3, pp. 581-597, March 2010

11. S. M. Hosseini, A. Johari, and A. Keshavarz, "Reliability Analysis of Seismic Bearing Capacity of Strip Footing by Stochastic Slip Lines Method," Computers \& Geotechnics, Vol. 91, pp. 203-217, 2017

12. C. Nan and D. Zhu, "Reliability Analysis of the High-Speed Train Bearing based on Wiener Process," Information, Vol. 9 , No. 1, pp. 15-17, January 2018

13. C. Tao, H. Ji, J. Qiu, Z. Wang, W. Yao, and C. Zhang, "Characterization of Fatigue Damages in Composite Laminates using Lamb Wave Velocity and Prediction of Residual life," Composite Structures, Vol. 166, pp. 219-228, 2017

14. D. Massih, A. H. Soubra, and B. K. Low, "Reliability-based Analysis and Design of Strip Footings Against Bearing Capacity Failure," Journal of Geotechnical \& Geoenvironmental Engineering, Vol. 134, No. 7, pp. 917-928, July 2011

15. R. J. Bathurst and N. Luo, "Reliability Bearing Capacity Analysis of Footings on Cohesive Soil Slopes using RFEM," Computers \& Geotechnics, Vol. 89, pp. 203-212, 2017

16. J. Chen, Y. Feng, and X. Huang, "Reliability-based Residual Life Prediction of Large-Size Low-Speed Slewing Bearings," Mechanism \& Machine Theory, Vol. 81, No. 11, pp. 94-106, November 2014

17. B. K. Low, D. S. Y. A. Massih, and A. H. Soubra, "Reliability-based Analysis and Design of Strip Footings against Bearing Capacity Failure," Journal of Geotechnical \& Geoenvironmental Engineering, Vol. 134, No. 7, pp. 917-928, July 2008

Han Zhang is a Master's student at Inner Mongolia University of Technology. His main research areas are equipment reliability and fault diagnosis.

Jianxin Wu is a professor and graduate student tutor at Inner Mongolia University of Technology. His main research areas are artificial intelligence, mechanical dynamics analysis and optimization, equipment reliability, and fault diagnosis.

Yiqing Qiu is a Master's student at Inner Mongolia University of Technology. His main research areas are mechanical dynamics analysis and optimization.

Qiang Chen is a Master's student at Inner Mongolia University of Technology. His main research areas are image processing and intelligent control technology. 